\title{
Civilisations
}

Revue internationale d'anthropologie et de sciences

humaines

60-1|2011

Mobilisations et dynamiques identitaires en Amérique andine

\section{Amitiés, inimitiés}

Les rapports incertains de l'Église catholique avec la Première

République voltaïque (1960-1966)

Jean-Marie Bouron

\section{(2) OpenEdition}

\section{Journals}

Édition électronique

URL : http://journals.openedition.org/civilisations/2763

DOI : 10.4000/civilisations. 2763

ISSN : 2032-0442

Éditeur

Institut de sociologie de l'Université Libre de Bruxelles

\section{Édition imprimée}

Date de publication : 31 décembre 2011

Pagination : $123-142$

ISBN : 2-87263-035-X

ISSN : 0009-8140

Référence électronique

Jean-Marie Bouron, «Amitiés, inimitiés », Civilisations [En ligne], 60-1 | 2011, mis en ligne le 15 février 2015, consulté le 02 mai 2019. URL : http://journals.openedition.org/civilisations/2763 ; DOI :

10.4000 /civilisations. 2763 


\title{
Amitiés, inimitiés \\ Les rapports incertains de l'Église catholique \\ avec la Première République voltaïque (1960-1966)
}

\author{
Jean-Marie BOURON
}

Résumé : La mémoire populaire a gardé des relations unissant l'Église catholique au régime de Maurice Yaméogo, premier président de la République de Haute-Volta, un double souvenir : celui d'une franche collaboration qui, peu avant la chute de la Première République, s'est dissoute lors d'un divorce soudain. Cet article, en recensant les différents modes d'intervention des acteurs catholiques de l'époque dans l'espace public, tente de revenir sur cette vision au premier abord trop simpliste. Pour cela, il convient de distinguer les relations officielles des réalités officieuses, de faire émerger différents contextes, d'analyser les manières de faire de la politique "par le haut " et "par le bas » et de tenir compte de la diversité du milieu catholique. Car bien que l'Église locale revendique une image uniforme et globalisante, ses membres, en fonction de la position qu'ils occupent et des différentes temporalités, interviennent dans le champ politique selon des modalités variables.

Mots-clés : Église catholique, espace public, pratiques politiques, Maurice Yaméogo, Haute-Volta.

Abstract: The popular memory has kept two things from the relations linking the Catholic Church to the regime of Maurice Yaméogo, first president of the Republic of Haute-Volta: a frank collaboration that collapsed in a sudden divorce, just before the fall of the first republic. This article, in listing the different modes of intervention of the catholic actors in the public space, tries to go back over this vision which is at a first look, too simplistic. So, it is advisable to distinguish the official from the unofficial relations, to highlight the different contexts, to analyse the ways of making politics on a "high" and on a "low" level in the catholic environment and to take into account the diversity in the catholic environment. Because, though the local church proclaims a uniform and a allembracing image, its members, according to the position they have and according to the different temporalities, intervene in the political field, following variable modalities.

Keywords: Catholic Church, public sphere, political practices, Maurice Yameogo, Upper-Volta. 
L 5 août 1960, la République voltaïque proclame son indépendance au son de La Volta, hymne national composé par l'abbé Robert Ouédraogo. La veille, Jean XXIII assurait déjà à cette jeune nation sa " large bénédiction $»^{1}$ après que Maurice Yaméogo, premier président de la Haute-Volta, ait recommandé au pape « tout un pays qui vous aime et vous vénère $»^{2}$. La célébration de l'indépendance résonne ainsi en suivant la douce mélodie d'une fine collaboration entre la nouvelle administration et l'Église catholique voltaïque, toutes deux souveraines de fraîche date. Mais au-delà de cet « effet d'oracle, grâce auquel le porte-parole fait parler le groupe au nom duquel il parle » (Bourdieu 2001 : 269), les relations entretenues par le milieu catholique - dans toute sa diversité - avec le champ politique sont bien souvent guidées par des réalités tacites, au point que leur perception devienne équivoque, voire insaisissable.

Comme le préconisent G. André et M. Hilgers, l'analyse parallèle de l'espace public et du « champ théologique » doit prendre en compte la « segmentarisation » du religieux (correspondant pour nous à l'« épaisseur » du milieu catholique). Ces auteurs, reprenant l'analyse bourdieusienne, s'interrogent alors sur " une possible correspondance entre la position sociale, la production et la réception d'une idéologie religieuse » (André et Hilgers 2009 : 11-12). Rapporté à notre sujet, ce questionnement suscite une nouvelle interrogation. L'étude des relations ecclésio-étatiques qui s'organisent en Haute-Volta entre 1960 et 1966 sera l'occasion de détecter ce qui, dans les rapports du religieux au politique, relève non seulement de la pluralité des acteurs du corps catholique mais également d'une conjoncture fluctuante et étagée. En d'autres termes, comment se conjuguent la diversité du milieu ecclésial et les lois du contexte dans la définition des comportements politicoreligieux?

Afin de mettre en évidence les multiples facettes des relations ecclésio-étatiques qui tantôt s'éclairent à la lumière des rapports officiels, tantôt se dissimulent derrière le paravent de l'implicite, nous devons croiser l'analyse chronologique et l'étude différenciée des acteurs catholiques. L'observation des rapports entre le président Yaméogo et le chef de l'Église voltaïque, Mgr Zoungrana, s'avère dans un premier temps essentielle. Car bien que personnifiées, ces relations sont investies du capital d'autorité indispensable pour créditer le dialogue ecclésio-étatique de la légitimité nécessaire. Cependant, les rapports entre l'État et l'Église ne sont pas uniquement incarnés par cette représentation bicéphale. Autant le parti unique n'est pas une entité homogène, autant la structure ecclésiale, malgré son allure bureaucratique, ne prononce un discours monocorde ni ne produit une activité monolithique. Au regard horizontal proposé par l'exposé événementiel, il devient donc nécessaire d'ajouter une translation verticale pour distinguer les actions engagées entre hiérarchies civiles et religieuses des multiples manières de faire de la politique «par le bas » (Bayart 1981). C'est ainsi que seront découvertes les logiques qui poussent l'Église et ses mandataires pendant la période considérée ici (1960-1966) à naviguer entre collaboration officielle, crispations dissimulées et opposition assumée.

1. Télégramme de Jean XXIII à Maurice Yaméogo, 4 août 1960, Archives de l'Archevêché de Ouagadougou (AAO), N111.

2. Télégramme de Maurice Yaméogo à Jean XXIII, Ouagadougou, 28 juillet 1960, AAO, N111. 


\section{Collaboration ou compromission, humanisme ou pragmatisme?}

\section{Une vision politique théocratique}

De la même façon que la Haute-Volta indépendante hérite des configurations coloniales, l'Église catholique devient légataire de son passé missionnaire. Le catholicisme, implanté à partir de 1900 par les Pères Blancs, se doit de participer durant la colonisation au champ politique pour satisfaire ses ambitions apostoliques. Ses rapports avec l'administration française se distinguent par leur inconstance et leur versatilité (de Benoist 1987). Nous découvrons toutefois au lendemain de la Seconde Guerre mondiale une mission catholique solidement installée au cœur du jeu colonial (Bouron 2010a). Les œuvres scolaires, une des pierres angulaires de la pastorale catholique, donnent alors l'occasion aux Pères et aux Sœurs de former les personnalités qui tiendront plus tard les rênes du pays. Mais après avoir « fabriqué » les élites du territoire, les missionnaires se retrouvent contraints de prendre en compte les aspirations de leurs anciens élèves. Évitant une immixtion trop prompte dans le milieu politique, ils cherchent à canaliser le mouvement qui mènera à l'indépendance par l'entremise de circuits détournés, notamment sur la base d'un discours consensuel fondé sur la doctrine sociale de l'Église (Bouron 2010b). Dans le même temps, l'africanisation des structures ecclésiales est en œuvre. Après la nomination d'un premier évêque voltaïque en 1956, la Haute-Volta célèbre son premier archevêque autochtone, Mgr Zoungrana, en 1960. Avec la fondation d'une Église locale, l'autorité catholique conserve toute sa légitimité en matière politique : elle se veut alors l'interlocutrice privilégiée d'un pouvoir qui réclame également sa souveraineté. En ces temps d'indépendance, la hiérarchie religieuse construit un discours nationalistes révélateur des prétentions ecclésiales :

\section{Toutes ces régions qui forment le pays de Haute-Volta sont nées à la Grâce du Christ sous le patronage de Marie Immaculée, lorsque nos premiers missionnaires lui consacrèrent ce champ de leur apostolat. Et c'est encore par une délicate attention de la Providence que la république de Haute-Volta est née sous le signe de Marie Immaculée, le 11 décembre en l'octave de sa fête 3 .}

Alors que la construction nationale peine à effacer les divergences régionales (Beucher 2009), le christianisme tente de devenir le dénominateur commun de la nation. En quête de légitimité, les ecclésiastiques proposent aux Voltaïques une filiation catholique qui, à l'aune de la composition sociologique du territoire, peut paraître contradictoire. Car faut-il le rappeler, la Haute-Volta abrite davantage de musulmans que de chrétiens, tandis que la religion traditionnelle domine encore à cette époque le paysage multiconfessionnel ${ }^{4}$. Aussi, la politique apparaît comme un tremplin assurant crédit et hégémonie au catholicisme. Electron libre voguant au sein d'un paysage sociopolitique constitué par l'aristocratie coutumière, les élites lettrées, les personnalités politiques et syndicales mais également des figures locales telles que les catéchistes (Bouron 2010c) ou les anciens combattants, l’Église recherche avant tout à dépasser sa situation sociologique minoritaire au profit d'une visibilité dominante.

3. Lettre de Mgr Zoungrana à l'occasion des fêtes du premier anniversaire de l'Indépendance, Ouagadougou, 3 décembre 1961, Centre National des Archives du Burkina Faso (CNABF), Cart. 6V16.

4. Les sources officielles font état de $27,5 \%$ de musulmans pour 3,8\% de chrétiens et $68,7 \%$ d'« animistes ». Enquête démographique par sondage en République de Haute-Volta 1960-1961, t. 1, p. 104. 
Dans ce but, la publication d'une lettre pastorale permet aux prélats voltaïques de s'accorder une prééminence morale qui déguise avec peine certains penchants théocratiques. Pour eux, « la source du pouvoir est en Dieu » (Les évêques de Haute-Volta 1959 : 27). Evangile et société étant imbriqués, la parole chrétienne doit enrichir l'action politique. Convaincue de son rôle, la hiérarchie catholique propose explicitement sa collaboration aux gouvernants : « En formant parmi ses fils des citoyens de cette qualité, croyez, Monsieur le Président, que l'Église, sans empiéter sur des compétences qui ne sont pas les siennes, est prête à vous apporter tout son concours pour la promotion de votre noble pays $»^{5}$. Maurice Yaméogo semble répondre positivement à cette proposition puisqu'il développe également le même type de rhétorique. Il ne s'en cache pas : la religion constitue l'assise sans laquelle « un commandement [est] voué à l'échec $»^{6}$, rappelant ainsi curieusement la légitimation religieuse de l'autorité moaaga ${ }^{7}$. Conceptions traditionnelle, catholique et présidentielle semblent alors converger vers une même définition de l'autorité.

\section{Dérives autocratiques et silence catholique}

Pourtant, ces considérations cléricales ne semblent pas trouver un écho suffisant pour organiser une véritable éthique politique catholique. Malgré quelques réactions internes, l'Église locale reste muette devant le durcissement du régime Yaméogo. Après une manipulation de la loi électorale, le parti unique devient effectif sous le sigle de l'UDV/RDA (Union Démocratique Voltaïque, section nationale du RDA). Libérée de toute opposition parlementaire, l'Assemblée nationale adopte en novembre 1960 une Constitution qui consacre le chef de l'État au sein d'un régime présidentiel disposant de pouvoirs élargis. Après avoir provoqué le ralliement de plusieurs ténors politiques, le président de la République marginalise les personnalités restées fidèles à l'opposition. Les plus chanceux, nommés ambassadeurs, bénéficient d'un exil doré. Pour les autres, les mesures étatiques sont plus expéditives. Par exemple, le 3 juillet 1960, au moment de l'installation à Ouagadougou du nouvel archevêque Mgr Zoungrana, deux personnes de l'opposition sont appréhendées par les forces de l'ordre ${ }^{8}$. Les cérémonies consacrant le premier métropolitain voltaïque restent alors entachées par l'application de la stratégie autoritaire du pouvoir en place. Pourtant, aucune réaction officielle de la hiérarchie catholique ne vient condamner ces mesures policières. Une intervention immédiate risquerait de mettre à mal la collaboration qui s'institue avec faste au moment de l'installation du nouveau prélat. Sans doute est-il plus profitable pour la hiérarchie de refouler les sentiments humanistes de certains chrétiens plutôt que de compromettre son avenir dans la sphère publique. C'est donc plusieurs mois plus tard que l'archevêque fraîchement nommé se décide à s'adresser au président de la République :

5. Allocution de Mgr Maury, Ouagadougou, 27 juin 1964, Archives Provinciales des Missionnaires d'Afrique, Paris (APMAfr), non classé.

6. Carrefour Africain 45, 18 février 1962.

7. Les Moose (sing. Moaaga), constituant la population majoritaire de la Haute-Volta, utilisent le concept de naam pour légitimer le pouvoir que leurs chefs reçoivent de Dieu.

8. Fiche de renseignement, Ministère de l'Intérieur, 4 juillet 1960, CNABF, Cart. 6V31. 
Le devoir m'oblige à vous en écrire à titre purement personnel, comme un ami parle à son ami. Ne pourraient-ils pas être relâchés, noblement, avant les élections (...)? Cette mesure de clémence donnerait au Gouvernement encore plus d'autorité et de force vis$\grave{a}$-vis des populations ; la confiance deviendrait plus réelle parce que plus spontanée. Il est bien évident que (...) vous ne pouvez pas tolérer des saboteurs de l'œuvre nationale que vous avez entreprise, et toute démarche équivoque de leur part pourrait vous conduire à des mesures encore plus énergiques que celles qui furent déjà prises?.

Sont-ce les devoirs de la diplomatie ou les relations amicales qui obligent la rhétorique de Mgr Zoungrana à privilégier la stratégie politique au discours humaniste ? En effet, lorsque le nouvel archevêque s'adresse à Yaméogo c'est pour mettre en exergue le bénéfice que pourrait retirer le président de la République de son éventuelle magnanimité. Il ne s'agit pas de s'offusquer des atteintes à la liberté mais bien d'envisager, si les « saboteurs » demeurent réfractaires, "des mesures plus énergiques » que les internements administratifs. On s'attendait à lire les lignes d'un défenseur des droits de l'Homme, on se surprend à découvrir une sorte d'éminence grise. Toujours est-il que l'argumentaire développé par Mgr Zoungrana semble convaincre Maurice Yaméogo puisque ce dernier apporte dans sa réponse la nouvelle de la libération des opposants ${ }^{10}$.

Lors de la campagne électorale pour les municipales, en 1960, l'Église catholique retrouve un discours en faveur des droits fondamentaux : "Nous ne pouvons vous cacher, bien chers Frères, que nous avons été peiné d'entendre dire que dans notre ville archiépiscopale de Ouagadougou, la liberté d'expression n'aurait pas toujours été respectée pendant la préparation des élections, et cela, peut-être à l'insu des Pouvoirs établis » $(\text { sic })^{11}$. Il est important de remarquer que ces propos tenus en chaire prennent soin de ne pas indexer le régime de Yaméogo, comme si l'autorité ecclésiastique souhaitait s'aménager une place aux côtés du pouvoir civil plutôt que de fragiliser sa position en adoptant un discours antagoniste. Ainsi, pour le clergé voltaïque, le pragmatisme politique dominerait l'humanisme chrétien. Maintenant que la stratégie de formation des élites récompense les Pères Blancs et les prêtres diocésains qui voient désormais des chrétiens occuper le devant de la scène publique, il ne leur est pas bienvenu de critiquer leurs anciens élèves.

\section{Repasser le linge propre en famille}

Des liens unissant le clergé à certains dirigeants issus du milieu missionnaire découle une certaine familiarité qui peut expliquer, en partie, le mutisme officiel de l'Église devant certains abus perpétrés par le pouvoir en place. Ainsi, c'est un ton réellement amical qui guide les relations entre Mgr Zoungrana et le président de la Haute-Volta. D’ailleurs, Maurice Yaméogo ne s'est-il pas, dans un premier temps, destiné à la prêtrise ? Élève du petit séminaire de Pabré au cours des années 1930, il y affiche sa solide conviction pour le sacerdoce (Yaméogo 1938 : 164). Finalement, il en sortira sans diplôme mais gardera le souvenir de ses anciens camarades : les abbés Robert Ouédraogo, Georges Yaogho, André-Jules Bouncoungou mais

9. Mgr Paul Zoungrana à Maurice Yaméogo, Ouagadougou, 10 octobre 1960, CNABF, Cart. 6V16.

10. Maurice Yaméogo à Mgr Zoungrana, Ouagadougou, 5 novembre 1960, CNABF, Cart. 6V16.

11. « Note de Mgr Zoungrana à lire en chaire dans toutes les églises de la ville de Ouagadougou le dimanche 18 décembre $1960 »$, CNABF, Cart. 6V16. 
aussi des anciens séminaristes qui, comme lui, se sont trouvés une vocation politique : Pierre Tapsoba, Joseph Ki-Zerbo, Joseph Ouédraogo (Guirma 1991 : 26-27). Même s'il préfère embrasser la carrière de fonctionnaire et ainsi profiter du prestige que lui confère son statut d' " évolué », Maurice Yaméogo continue de côtoyer les membres d'une Église en cours d'africanisation. Il fait appel, par exemple, au Père Paul Zoungrana pour baptiser son fils ${ }^{12}$. C'est d'ailleurs à cette occasion que le prêtre apprend la nouvelle de sa nomination à la charge épiscopale. Le président de la République voltaïque devient ainsi le témoin direct de l'africanisation de l'archevêché de Ouagadougou. Les deux hommes accèdent ainsi simultanément aux plus hautes responsabilités que leur assurent leurs vocations respectives. Cette occurrence renforce leur fraternité. Les rapports entre l'archevêque de Ouagadougou et l'ancien séminariste semblent ainsi révélateurs d'une évidente familiarité entre l'appareil d'État et les structures ecclésiales.

Accouchant à la fois du clergé africain et de l'élite politique, les œuvres scolaires missionnaires ont fait des rapports ecclésio-étatiques des relations de consanguinité. $\mathrm{Au}$ premier sens du terme tout d'abord, il n'est pas rare de constater dans une même famille un prêtre côtoyant son frère haut fonctionnaire ${ }^{13}$. À un niveau plus large ensuite, les écoles catholiques forment côte à côte les ecclésiastiques et les futurs dirigeants. Certaines relations nouées pendant l'enfance se pérennisent par des liens de cooptation. Par exemple, Gabriel et Alexandre Zoungrana, cadets de l'archevêque et anciens séminaristes tout comme Yaméogo, trouvent dans le chef de l'État voltaïque un appui confortable pour relancer leur carrière ${ }^{14}$. Juste retour des choses, Mgr Zoungrana se démène pour trouver une place dans un collège jésuite pour le fils d'un député15.

La proximité qui unit le clergé aux hommes politiques apparaît également pour certains ecclésiastiques comme une situation profitable. Ainsi, au-delà des intérêts pastoraux, certains prêtres cherchent également dans la fréquentation du monde politique des acquis temporels permettant d'achever leur promotion sociale. Tel, par exemple, cet abbé qui, dans une familiarité déconcertante, demande à « Maurice » une voiture pour faciliter son ministère et, on s'en doute, accroître son prestige ${ }^{16}$. Sollicité, le chef de l'État profite de l'inauguration du palais qu'il a fait construire dans sa ville natale de Koudougou pour répondre à la requête du prêtre. Ce dernier, en tant que « camarade d'enfance » ${ }^{17}$, vient bénir l'immense villa. Il reçoit alors, comme « droit d'étole », une 2 CV neuve (Bouncoungou 1971 : 153).

Représentée officiellement au moment de l'inauguration du palais de Yaméogo, la hiérarchie catholique donne l'image d'une autorité compromise, s'affichant aux côtés d'un régime qui par ses frasques fait du luxe sa raison d'être. Et même si l'intention première du

12. Mgr Zoungrana au Supérieur général Volker, Ouagadougou, 12 avril 1960, AGMAfr, Dos. 683 (I).

13. Un exemple : André Balkouma, commandant de cercle puis chef de cabinet du ministère de l'Intérieur en 1963, est le frère de l'abbé François. Diaire de Zorgho, 25 juin 1962, AGMAfr.

14. Gabriel, après avoir fait de la prison, est mis au service du gouvernement voltaïque pendant que Maurice Yaméogo trouve auprès de l'ambassade de France au Vatican une formation en diplomatie pour Alexandre (Guirma 1991 : 121).

15. Mgr Zoungrana au R.P. Becquart, Ouagadougou, 27 août 1960, APMAfr, Dos. 211.

16. Abbé André-Jules Bouncoungou à Maurice Yaméogo, Ouagadougou, 17 octobre 1960, CNABF, Cart. 6V16.

17. Afrique Nouvelle 882, 3 au 9 juillet 1964. 
président de la République n'est pas ici de corrompre l'Église, il reste difficile de ne pas reprendre, avec Jean-François Bayart, l'expression qui suggère que « la bouche qui mange ne parle pas », formule bienheureuse pour décrire la « gouvernementalité du ventre » des Églises africaines (Bayart 1993 : 145). Avec l'indépendance, les besoins financiers se font de plus en plus sentir pour une institution ecclésiale en pleine expansion. Il s'agit alors pour certains prêtres de s'intégrer aux logiques clientélistes entretenues par les proches du pouvoir pour que l'Église puisse elle aussi « émarger (...) aux prébendes redistribuées par les princes des indépendances africaines » (Mbembe 1988 : 176). À leur tour, ces configurations patrimoniales se reproduisent au sein de l'institution ecclésiale où s'observent difficultés de gestion et constitution d'une classe de prêtres privilégiés (Bayart 1989 : 1). Ce sont alors généralement les ecclésiastiques les mieux pourvus en capitaux sociaux et symboliques qui accèdent avec le plus d'aisance à l'économie de captation. Il y a lieu ici de distinguer les prêtres des paroisses rurales qui ne peuvent profiter avec la même célérité de la redistribution étatique, des ecclésiastiques bourgeois, souvent diplômés et se comportant parfois comme de véritables « entrepreneurs ».

Le pouvoir se fait alors remarquer par son évergétisme. Maurice Yaméogo n'oublie pas ses origines. De la même manière qu'il multiplie les efforts pour développer sa ville natale, il donne également la priorité au petit séminaire de Pabré pour le faire profiter des avantages du service public. De nombreux dons étatiques sont alloués à la construction d'édifices religieux ou pour le confort du personnel ecclésiastique ${ }^{18}$. Le comble de cette munificence présidentielle est atteint avec le projet d'édification, à l'instigation de Maurice Yaméogo, d'une nouvelle cathédrale à Ouagadougou ${ }^{19}$. Volonté est ici affichée de se concilier l'appui catholique. En retour des générosités étatiques, le président de la République s'attend à un contre-don bien moins matériel : la collaboration de la hiérarchie catholique. Garante d'une partie de l'ordre social - puisque dépositaire d'un ordre symbolique -, l'Église est également un facteur de légitimation pour le pouvoir politique (Bourdieu 1971 : 328). Ainsi, aux marges de l'organigramme officiel, les autorités catholiques sont invitées à prendre part au secteur « non-structuré » de l'État, lieu où se rencontrent « l'institutionnel et l'informel, le politique et l'économique, l'affectivité (les liens d'origine ou de confession) et la rationalité (les avantages recherchés) » (Martin $1991:$ 164).

\section{Festivités}

Ainsi, les autorités religieuses se retrouvent régulièrement associées aux cérémonies célébrant le nouvel État indépendant. L'occasion est alors offerte à l'Église locale d'exprimer son patriotisme ${ }^{20}$. En retour, Yaméogo mobilise le protocole étatique pour célébrer les grandes dates de l'Église du pays. Ainsi, lors du sacre à Rome de Mgr Zoungrana, le président de la République fait le déplacement et finance le voyage de plusieurs personnalités religieuses et politiques $^{21}$. Malgré les arrestations précédemment évoquées, le nouveau prélat, lors de son installation à Ouagadougou se retrouve encore aux côtés de Yaméogo :

18. Voir CNABF, Cart. 7V484 et Cart. 6V16.

19. Mgr Zoungrana à Maurice Yaméogo, Ouagadougou, 6 mai 1961, CNABF, Cart. 6 V16.

20. Mgr Zoungrana à l'occasion des fêtes du premier anniversaire de l'Indépendance, doc. cit.

21. P. Grimault au Supérieur général Volker, Ouagadougou, 27 avril 1960, AGMAfr, Dos. 683 (I). 


\begin{abstract}
Monseigneur l'Archevêque, Monsieur le Président de la République et Monseigneur le Délégué [apostolique] se mirent à haranguer la foule tour à tour pour parler de la collaboration des deux pouvoirs. On peut se demander lequel des trois a été le moins digne des Pères de l'Église. Que Dieu fasse que tous les serments soient autre chose que des paroles ${ }^{22}$.
\end{abstract}

Le vœu du rédacteur de cette note semble se réaliser en 1965, lors du retour en Haute-Volta de l'archevêque de Ouagadougou récemment nommé cardinal. Attachons-nous à relever le parallélisme avec les grandes cérémonies civiles :

À sa descente de l'avion, le Cardinal Zoungrana tout de rouge vêtu a été accueilli par les Chefs d'État de Côte d'Ivoire, du Dahomey et de Haute-Volta entourés de nombreuses personnalités. Après l'exécution des hymnes ivoirien, dahoméen et voltaïque, le Ministre du pape a passé en revue le détachement de l'Armée nationale qui rendait les honneurs et a salué toutes les Délégations. Ayant pris ensuite place dans une voiture découverte aux côtés des Chefs d'État, le Cardinal Zoungrana a gagné l'Archevêché acclamé tout au long du parcours par une foule immense et enthousiaste. Dans la vaste cour de l'Archevêché pavoisée aux couleurs vaticanes et voltaïques, une foule nombreuse de fidèles, nullement inquiétés par la chaleur, manifestait sa joie par des danses folkloriques et poussait par instants des hourras enthousiastes dès que le Cardinal Zoungrana fit son entrée à la Mission Catholique, d'abord salué par des salves de coups de feu. Après cet instant solennel, le Ministre du pape devait prendre place sur la tribune officielle aux côtés des Présidents [membres du Conseil de l'Entente] ${ }^{23}$.

Puis s'ensuit l'allocution du président ivoirien qui prend soin de voir dans la conjugaison entre l'action spirituelle et l'activité temporelle de Mgr Zoungrana l'explication de son accession au cardinalat. Proche du sermon, ce discours, en citant l'Evangile, rappelle la récupération par la classe politique de symboles et de concepts religieux, désormais mobilisés par certains dirigeants africains (Rivière 1997 : 30). Plus troublant encore est de surprendre une autorité catholique - si éminente soit-elle - en train de se mouvoir avec facilité et sans travestissement dans un décor réservé habituellement aux plus hautes personnalités politiques. Le parallèle est patent au point que Bayart classe les solennités religieuses «parmi les principaux rituels sociaux de l'Afrique postcoloniale, de pair avec les manifestations de masse des partis uniques, les cérémonies d'intronisation de quelques souverains prestigieux, les matchs de football et les exécutions capitales » (Bayart 1993 : 139). Paroxysme de l'emprunt par la puissance ecclésiastique des symboles du pouvoir institutionnel, une telle cérémonie fait en définitive du « Ministre du Pape » une figure qui tient davantage d'un culte temporel de la personnalité que d'une dévotion à l'adresse d'un intercesseur religieux.

L'interpénétration des codes protocolaires civils et des cérémonies spirituelles dévoile avant tout une situation officielle. On peut y déceler une volonté tant étatique qu'ecclésiastique de collaborer en vue d'un partage maximal du pouvoir. Néanmoins, derrière cet esprit de concorde peuvent se cacher d'autres dimensions bien moins perceptibles.

22. «Fêtes de l'intronisation de Monseigneur Zoungrana », Ouagadougou, 7 juillet 1960, AGMAfr, Dos. 683.

23. Compte-rendu de l'arrivée à Ouagadougou du Cardinal Paul Zoungrana, 3 mai 1965, CNABF, Cart. 7V484 . 


\section{Laïcité et autocensure}

L'intégration des élites sacerdotales au sein des logiques de l'État postcolonial ne doit pas être perçue comme la preuve de la corruptibilité de l'Église qui, au prétexte de subventions ou de relations amicales, multiplierait les révérences au régime. Si elle accepte de parader aux côtés du pouvoir c'est également pour en retirer un avantage apostolique. Ainsi, de la même manière que l'État postcolonial ne peut s'accorder le luxe de refuser l'appui catholique, les autorités ecclésiales ont besoin de la sollicitude du régime pour parfaire leur projet de christianisation. À une période charnière où les dynamiques de la politique nationale détournent de nombreux chrétiens de leur ferveur habituelle, l'Église craint de perdre son leadership moral sur la société voltaïque. Maurice Yaméogo se veut pourtant rassurant. Le chef de l'État voltaïque, lors de sa visite officielle au Vatican en 1962, a d'ailleurs désigné le pays qu'il représente comme « une fille aînée de l'Église sur la terre d'Afrique $»^{24}$. Mgr Dupont, évêque de Bobo-Dioulasso l'avoue également : le « catholicisme pouvait paraître, à certains jours, comme la Religion d'État» (Dupont s.d. : 30). Et de fait, pour la majorité des témoins de l'époque, à l'instar de ce pasteur des Assemblées de Dieu, « le gouvernement est catholique et le catholicisme dispose ainsi d'une citadelle dans le pays $»^{25}$.

Néanmoins, la demeure abritant autorités civiles et religieuses ressemble parfois à un décor en carton-pâte. Car autant l'identité confessionnelle du régime s'affiche ostensiblement, autant les nécessités de la construction nationale limitent toute cléricalisation du pouvoir. En effet, c'est l'idéologie de la laïcité qui semble dominer la période d'indépendance. Il s'agit pour l'État en construction de réaliser « un travail sur le religieux » (Coulon 1991 : 9596) pour le contrôler et présider seul aux destinées nationales. Même si une attitude parfois fusionnelle est mise en avant, ambitions étatiques et projets ecclésiastiques ne peuvent se confondre. L'évocation explicite des règles de la laïcité constatée à plusieurs reprises sous la plume de Yaméogo ${ }^{26}$ n'est-elle pas le moyen de rappeler aux forces religieuses que leur rôle en matière politique se résume aux applaudissements devant les défilés du parti unique ou au silence face aux dérives autocratiques ? Amer, Mgr Zoungrana est contraint de déplorer l'incohérence des dirigeants voltaïques, pourtant chrétiens pour la plupart : « Les hommes peuvent bien élaborer une Constitution, mais ils ne peuvent élaborer un code de morale qu'ils appelleront "Commandements de Dieu" $\gg{ }^{27}$. Mais les Livres de loi ne sont pas les mêmes. Les intérêts non plus.

Pour éviter les travers du cléricalisme, la hiérarchie catholique serait ainsi invitée à s'autocensurer et à s'investir uniquement autour des questions théologiques. Le contexte en donne le prétexte. En effet, réuni entre 1962 et 1965, Vatican II concentre l'attention du clergé voltaïque. Les principaux évêques du pays siègent au sein des commissions conciliaires et le clergé dans son ensemble se montre mobilisé pour échafauder une théologie de l'inculturation. Aussi n'est-il pas étonnant qu'à cette époque les lettres pastorales des évêques africains

24. La Croix, 27 avril 1962.

25. Curtis L. Dean to Wendall L. Rockey, Wichita, December 27, 1962, Assemblies of God Archives, Springfield (AGAS), non classé.

26. Lettre de Maurice Yaméogo à Mgr Zoungrana, Ouagadougou, octobre 1961, ANBF, Cart. 6V16

27. Rapport annuel 1960-1961 de l'archevêché de Ouagadougou, doc. cit. : 2. 
se concentrent presque exclusivement autour d'enjeux ecclésiaux (Djereke 2001 : 34). Obnubilée par la réforme des stratégies missionnaires développées durant la période coloniale, l'Église catholique en oublie de faire la critique des configurations héritées des indépendances.

Dans le diocèse de Bobo-Dioulasso, le clergé préfère ainsi respecter scrupuleusement les principes du laïcisme gouvernemental. Puisqu'il « ne s'engage pas sur le terrain politique $»^{28}$, il peut conserver son autonomie. Encore dirigée par un évêque européen et éloignée de la capitale et du plateau moaaga, la circonscription de Bobo-Dioulasso semble hériter de la période coloniale un apolitisme de circonstance (Bouron 2010a : 78). Nous découvrons ainsi que l'implication des agents religieux dans l'espace public ne revêt pas la même intensité en fonction de variantes régionales. Plus que cela, l'Église voltaïque reproduit certaines divisions qui tourmentent déjà l'unité nationale. En particulier, la prédominance des Moose dans le clergé local est considérée par certains cadres comme un nouvel hégémonisme. Au grand séminaire, on redoute particulièrement la transposition au sein des structures ecclésiales d'une prétendue «domination moaaga » déjà constatée dans l'espace politique traditionnel et moderne ${ }^{29}$.

\section{Limites de l'État postcolonial et contours de la contestation catholique}

\section{Les prémices d'une opposition}

Lorsqu'il évoque les rapports entre l'Église et l'État voltaïques, René Otayek situe la première vexation en 1964 au moment où le gouvernement se permet de réorganiser le régime des subventions allouées à l'enseignement privé (Otayek 1997 : 230). Une première décision, en mars 1963, avait déjà supprimé une partie des contributions gouvernementales (Compaoré 1995 : 317). Mais en janvier 1964, le coup semble fatal pour les œuvres scolaires catholiques. Les subsides publics étant désormais distribués d'une manière aléatoire, l'Église voltaïque n'a d'autre choix que de creuser son déficit pour payer le salaire de ses enseignants. Finalement, elle sera contrainte cinq ans plus tard de céder une partie de ses structures éducatives à la tutelle étatique. Certains cadres du parti unique trouvent dans ces décrets le moyen d'éprouver la prééminence catholique ${ }^{30}$. Le camouflet gouvernemental dessine donc de sombres perspectives pour la visibilité catholique. Le préjudice est tel que la limitation des subsides de l'État devient le prétexte de la rupture du pacte de réciprocité précédemment évoqué. La politique du « donnant-donnant » n'ayant plus sa raison d'être, les critiques du régime risquent de commencer à se faire entendre dans la bouche de la hiérarchie catholique.

Toutefois, certains prêtres ne semblent pas avoir attendu cet épisode malheureux pour désavouer les choix du pouvoir en place. La différence réside dans la visibilité du désaccord: tandis qu'après 1964 la posture contestataire de l'Église apparaît clairement, les manifestations de désaccord des premières années du régime Yaméogo demeurent feutrées. Les premiers signes de tension sont repérables aussitôt l'indépendance proclamée. Le jeune président de

28. Rapport annuel 1958-1959 du diocèse de Bobo-Dioulasso, AGMAfr, Dos. 680 : 2.

29. Entretien avec l'abbé Abel Ouédraogo, Ouagadougou, 26 février 2011.

30. «Quelques échos environnant le discours du Président de la République à Nouna », 1964, APMAfr, non classé. 
la République, en même temps qu'il installe son régime par des mesures autoritaires, tente d'exercer un véritable contrôle sur certaines œuvres missionnaires. Cette tendance s'exprime par exemple quand Yaméogo insiste auprès des Pères Blancs pour que l'Institut d'études sociales qu'ils envisagent de créer soit implanté à Ouagadougou et non à Bobo-Dioulasso. En pleine période de musellement de l'opposition, et connaissant la capacité émancipatrice de la formation missionnaire, le chef de l'État craint que l'autorité qui l'a enfanté spirituellement puisse devenir l'instrument de sa chute. Il s'agit en effet à travers ce centre de formation de constituer une élite chrétienne sensible au contexte sociopolitique du moment. Pour le rassurer, le Supérieur général des Pères Blancs demande la médiation de Mgr Zoungrana pour préciser qu'il « ne s'agit en aucune manière de former des révolutionnaires, des anarchistes $»^{31}$. Finalement, l'argumentaire épiscopal est suffisamment convaincant, et aujourd'hui encore le Centre d'Etudes Economiques et Sociales de l'Afrique de l'Ouest (CESAO) siège à BoboDioulasso.

Preuve que les premières vagues qui viennent troubler la mer d'huile des relations ecclésio-étatiques se forment dès la période de l'indépendance, Maurice Yaméogo cherche dès 1960 à contenir la houle contestataire en expulsant un missionnaire. Après des critiques antigouvernementales trop vertement exprimées, le Père Sainsaulieu fait ainsi les frais des restrictions de la liberté d'expression :

Je ne puis vous cacher que son âme chevaleresque qui le poussait à défendre les intérêts des paysans, lui fit commettre des écarts de langage contre un ministre qui lui épargna de justesse... les menottes! Il dut quitter le territoire. Il avait sans doute raison dans le fond de la question, la forme était moins que élégante! 32

Il est ici intéressant de remarquer que l'évêque responsable du Père indocile condamne moins le contenu des critiques du missionnaire que leur formulation. Autrement dit, le silence ou les signes d'allégeance de la hiérarchie catholique peuvent dissimuler des sentiments bien moins sympathiques. Mais les intérêts ecclésiaux du moment empêchent une prise de position trop franche et toute incartade doit paraître isolée. Aussi, les signes d'antagonisme sont davantage perceptibles chez certains membres du clergé, souvent engagés dans le domaine social (Gallibert 2010 : 299) ${ }^{33}$, qu'au sein de l'Église nationale.

\section{La jeunesse écartelée entre deux projets antagoniques}

Autre pan de la politique autoritaire du président Yaméogo, les mouvements de jeunesse proches du pouvoir ne semblent pas recevoir la bénédiction épiscopale (Les évêques de Haute-Volta 1959 : 24-25). Les autorités ecclésiastiques gardent le souvenir des dérives des régimes fascistes. Pourtant, c'est bien plus la concurrence avec les courants de l'Action catholique que souhaite contester l'Église. En effet, ces mouvements, dans une double optique d'évangélisation intensive et extensive, permettent de véhiculer l'idéal chrétien au sein de la société. Participant à forger une conscience politique conforme à la morale

31. Supérieur général Volker à Mgr Zoungrana, Rome, 9 décembre 1960, AGMAfr, Dos. 683 (I).

32. Mgr Bretault au P. Garitan, Bobo-Dioulasso, 14 avril 1961, APMAfr, Dos. 212.

33. Le P. Sainsaulieu était l'animateur d'un centre de formation agricole à Dionkélé. Nous relevons également le cas du P. Tryers qui fut expulsé du Ghana à la même époque et pour les mêmes raisons. Ce missionnaire était l'un des promoteurs du christianisme social à Tamale. Notice nécrologique du P. Tom Tryers. 
religieuse, l'Action catholique fait également partie de la mécanique électorale instituée par les missionnaires ${ }^{34}$. À la suite de mots d'ordre de prêtres, les jeunes peuvent notamment se déplacer en masse pour certaines élections. Un témoin évoque par exemple le cas d'un militant du Mouvement de Libération Nationale (MLN), parti alors contraint à la clandestinité, qui, au bénéfice du soutien de la paroisse locale, reste l'inamovible président de la Maison des Jeunes de Koudougou ${ }^{35}$. Alors qu'au niveau national c'est l'image d'un concordat entre le pouvoir politique et l'autorité religieuse qui prévaut, au niveau local on découvre certaines formes de contestations qui prennent source au sein de l'institution catholique. La jeunesse, cette force vive que l'Église et la nation tentent de contrôler, devient donc l'enjeu d'une lutte dissimulée. Nous sommes alors bien loin du temps où organisations confessionnelles et laïques tenaient un congrès commun à Ouagadougou ${ }^{36}$. Dès avant 1960, lorsque la Jeunesse Voltaïque du RDA se rassemble, des slogans anticléricaux peuvent être scandés ${ }^{37}$. Dès lors, la hiérarchie catholique considère l'encadrement du RDA comme un facteur de sécularisation et un vecteur d'immoralité. Simultanément, on voit Maurice Yaméogo s'appuyer sur la jeunesse pour instaurer le travail d'intérêt collectif. Pratiqué le dimanche, il remet en cause le principe du repos dominical. Suscitant alors l'hostilité des prêtres et des chrétiens - qui avaient déjà combattu l'administration coloniale sur ce point particulier -, cette mesure finit par être abrogée ${ }^{38}$.

Est-ce parce que l'Action catholique constitue une poche de contestation que sa suppression est envisagée par le régime en place ? En effet, les cadres du RDA constatent que les militants des organisations catholiques se désolidarisent trop facilement du parti unique. Au début des années 1960, la dissolution de l'Action catholique est très sérieusement évoquée ${ }^{39}$. D'ailleurs, le gouvernement voltaïque ne serait pas le premier à procéder à une telle mesure. Kwame Nkrumah, en rapports étroits avec la Haute-Volta au début des années 1960, a notamment substitué aux boys scouts un mouvement plus prompt à relayer l'idéologie du pouvoir et à entretenir le culte de sa personnalité (Gocking 2005 : 133). Avec ces mêmes ambitions, Mobutu supprimera lui aussi les mouvements confessionnels en 1972 (Nkay Malu 2003 : 305).

Voyant déjà ses écoles menacées, l'Église voltaïque ne peut laisser ses mouvements d'Action catholique succomber aux intentions du régime. Finalement, les organisations chrétiennes continuent d'exister mais certaines subissent les coups de boutoir du régime. Par exemple, après qu'un groupe de militants interpelle l'État pour mettre fin aux agissements de certains hommes politiques qui abusent de la liberté de la jeune fille, une trentaine de fonctionnaires est mutée. Ces sanctions ne concernent pas les personnalités aux principes

34. Voir Hastings 1979 : 99 pour le cas ouagandais.

35. Entretien avec Henri Guissou, Ouagadougou, 23 juin 2009.

36. Bulletin mensuel de politique générale, AOF, mai 1956, Centre des Archives d'Outre-Mer (CAOM), Cart. $2233: 4$.

37. Diaire de Koupéla, 5 août 1958, AGMAfr.

38. Entretien avec Norbert Ouédraogo, Ouagadougou, 3 juin 2009.

39. «Quelques échos environnant le discours du Président de la République à Nouna », doc. cit. 
douteux mais bien les auxiliaires de l'administration appartenant au mouvement catholique ${ }^{40}$. Malgré leur répression, les groupements chrétiens parviennent aux côtés des syndicats à contrebalancer l'hégémonie du régime. «Face au Parti-État lointain, les associations représentent la proximité (...), [lequel] délimite un espace-refuge, un espace d'autonomie à l'abri des exhortations du pouvoir, de ses mots d'ordre et ses campagnes de mobilisation » (Otayek 1991 : 189). Les possibilités de formation civique et de contestation offertes par les mouvements confessionnels offrent ainsi à l'Église le moyen de faire de la politique « par le bas ».

\section{Controverses localisées}

Au-delà du « domaine mixte $»^{41}$ que constitue la jeunesse, le comportement individuel de certains prêtres représente également une potentialité séditieuse pour le régime de Yaméogo. En effet, loin de la position officielle de l'épiscopat voltaïque, il semble qu'au sein de certaines paroisses des opinions antagoniques tendent à apporter la controverse au pouvoir en place. Le climat devient suspicieux au point que le chef de l'État commandite des " enquêtes secrètes $»^{42}$ pour jauger l'attitude de la classe ecclésiastique. En possession des résultats de ces investigations, il décide de convoquer Mgr Zoungrana pour le mettre en garde « sur l'atmosphère de subversion dont certains membres du Clergé seraient en partie responsables $»^{43}$. Parmi les griefs retenus contre le personnel ecclésiastique, Yaméogo reproche à certains membres de l'Église de soutenir l'opposition. Tandis que les paroisses de Kologh-Naba et de Dapoya font l'objet de soupçons du pouvoir après la distribution de tracts contestataires, certains prêtres sont vus comme les manipulateurs d'une jeunesse insoumise. Des aumôniers de l'Action catholique dénoncent les dépenses somptuaires de l'État. Également, des représentants de l'Église sont suspectés de soutenir Joseph Ouédraogo, éternel adversaire du président voltaïque. Nous savons par ailleurs que Mgrs Zoungrana, Yougbaré et Lesourd reçoivent une brochure antigouvernementale rédigée par Nazi Boni, opposant exilé au Sénégal ${ }^{44}$. Enfin, Yaméogo affirme avoir reçu une lettre menaçante après qu'il a limogé de ses charges ministérielles et coutumières François Bouda, personnalité proche des Pères Blancs. Ces différents crimes de lèse-majesté attirent ainsi la défiance du pouvoir politique à l'égard des actes que posent des membres de l'institution ecclésiale.

L'insoumission de certains membres du clergé légitime pleinement la réforme du régime des subventions consacrées aux écoles privées. C'est effectivement durant la même période que, en parallèle, s'affichent les signes d'hostilité de prêtres et que se multiplient les procès d'intention à leur encontre. Certains cadres optent pour une nouvelle saignée dans les subsides alloués aux établissements catholiques ${ }^{45}$. Mais le président de la République préfère

40. «Action Catholique des Familles de Nouna », mai 1964, APMAfr, non classé.

41. C'est Mgr Zoungrana qui, en s'adressant aux membres du gouvernement, évoque les « domaines mixtes » qui peuvent « amener des confrontations d'opinion, des divergences de vues ». Afrique Nouvelle, $\mathrm{n}^{\circ} 914, \mathrm{du}$ 4 au 10 février 1965.

42. Entrevue de Mgr l'Archevêque avec Mr. le président de la République, 6 janvier 1964, AAO, N111.

43. Ibidem.

44. Note de renseignement, Ministère de l'Intérieur, 15 octobre 1960, CNABF, Cart.6V25.

45. «Quelques échos environnant le discours du Président de la République à Nouna », doc. cit. 
explicitement « opérer tout ce qui pourrait devenir un cancer $»^{46}$. Effectivement, loin de la capitale, les signes d'insoumission se multiplient et un rappel à l'ordre s'impose. Dans le diocèse de Nouna, cinq missionnaires sont accusés de " trop parler » tandis que dans l'ensemble des communautés rurales, l'Église «"tambourinise” trop ses réalisations et les oppose à l'inertie du gouvernement $»^{47}$. Il est vrai que la faillite de l'État contraste parfois avec les initiatives développementalistes menées par le clergé. Pour étouffer les contestations, Maurice Yaméogo se déplace à Nouna où il définit clairement le rôle que l'État concède à l'Église : les hiérarchies religieuses doivent « se cantonner à essayer de sauver les âmes et non de risquer de faire brûler les corps » en allumant « une guerre religieuse, qui ne laisserait au pays d'autres alternatives que les excès sanglants des passions déchaînées $»^{48}$. Il menace alors de mesures de rétorsion ceux qui seraient tentés de déstabiliser le pouvoir. Qu'il soit prêtre ou homme politique, l'opposant n'aurait donc d'autre destin que la spoliation de ses biens, l'exil ou l'emprisonnement. La mise en garde paraît cinglante. D'autres cadres du parti, dont le président de l'Assemblée nationale, l'auraient souhaitée encore plus acerbe ${ }^{49}$. Finalement, après avoir mis en garde le milieu catholique contre toute immixtion dans les affaires de l'État, le président de la République y va de sa proposition pour réformer l'ambiance ecclésiale :

\section{Si les ingérences dans les affaires intérieures des autres mettaient permises, j'aurais vivement souhaité (...) qu'une africanisation des postes de responsables des Missions religieuses soit accélérée, notamment en brousse ; ainsi donc les difficultés que nous rencontrons aujourd 'hui dans différents domaines tomberaient du même coup, car nous aurions alors en face de nous des interlocuteurs valables du fait de la langue et même de la nationalité50.}

Le responsable des troubles semble ici tout désigné : c'est le prêtre étranger qui compromet l'œuvre nationale. Il est vrai que la parenté imaginée qui associe ecclésiastiques et dirigeants politiques semble moins étroite dans le milieu missionnaire qu'au sein des communautés d'abbés. En outre, les Pères Blancs ne sont pas concernés au même degré par les logiques clientélistes qui organisent nombre d'Églises africaines. C'est d'ailleurs par eux que transitent encore une partie des ressources diocésaines. Enfin, la pastorale de proximité que les missionnaires cherchent à entretenir les distingue de la bureaucratisation croissante de la hiérarchie catholique voltaïque. Les Pères et les Sœurs conservent par conséquent une autonomie et un contact avec les populations locales qui les éloignent de la sujétion au pouvoir en place. Disposant d'une liberté de ton qu'ils ont entretenue durant la période coloniale, les missionnaires semblent par contre oublier que la souveraineté a doublement changé de camp. D'une part, « certaines choses pouvaient se faire contre l'administration française, dans le passé, mais ne sont plus du tout de mise maintenant contre le pouvoir autochtone $»^{51}$. D'autre

46. Discours de Maurice Yaméogo à Nouna, 27 avril 1964, APMAfr, non classé.

47. «Quelques échos environnant le discours du Président de la République à Nouna », doc. cit.

48. Discours de Maurice Yaméogo à Nouna, doc. cit.

49. P. Tissier, « Attitude des milieux diplomatiques », 1964, APMAfr, non classé.

50. Discours de Maurice Yaméogo à Nouna, doc. cit.

51. P. Tissier, « Attitude des milieux diplomatiques », doc. cit. 
part, certains missionnaires européens font la douloureuse expérience de devoir obéir aux ordres du clergé diocésain qu'ils ont contribué à former (Somé 2001).

\section{Divorces}

Maurice Yaméogo tient donc à l'œil les prêtres, particulièrement les missionnaires étrangers. Pourtant, ce n'est pas du milieu ecclésiastique que va provenir la rupture. C'est bien le comportement de Yaméogo qui va déclencher les foudres de l'Église voltaïque et non l'inverse. En effet, après avoir reçu les preuves de l'adultère de sa femme, le chef de l'État voltaïque fait emprisonner cette dernière en compagnie de son amant. Or, ce ne sont pas tant les sévices infligés au couple infortuné que les conséquences de la répudiation qui provoquent l'ire de l'autorité catholique. Car aussitôt le divorce consommé, le président de la République se remarie avec une jeune ivoirienne en novembre 1965. Il n'en faut pas plus pour rompre la concorde officielle qui l'unissait à l'Église locale. Depuis Rome, où ils assistent au concile, les évêques voltaïques télégraphient leur désapprobation (Bouncoungou 1971 : 154). L'ensemble de la population s'offusque du comportement présidentiel, certains chrétiens allant jusqu'à réclamer l'excommunication du chef de l'État. Même dans l'équipe gouvernementale, certains ministres se disent « choqués $»^{52}$.

Touchée dans son for intérieur, l'Église catholique ne se cache plus pour montrer son mécontentement face aux pratiques du régime Yaméogo. Le remariage du chef de l'État, parce qu'il constitue la trahison morale de l'élite catholique, devient l'élément déclencheur de l'hostilité ecclésiale. Alors que sur d'autres points de discorde, le pouvoir politique et l'autorité religieuse préféraient maintenir l'image d'une amitié évidente, lorsque la plus haute personnalité de l'État bafoue le droit canonique, l'Église ne peut rester de marbre. Même s'il est crédité de 99\% des suffrages au moment de sa réélection en octobre 1965, Yaméogo doit faire face à une opinion publique de plus en plus irritée par sa politique autoritaire et ses caprices insolents. Tandis qu'il convole en noces lors d'un fastueux voyage en Amérique latine, le climat social se durcit. Le vent a tourné et l'autorité catholique le sait. Sachant, pour reprendre les mots de Pierre Bourdieu, que « la subversion symbolique de l'ordre symbolique ne peut affecter l'ordre politique que lorsqu'elle accompagne une subversion politique de cet ordre » (Bourdieu 1971 : 328), l'Église voltaïque trouve tous les ingrédients réunis pour faire entendre sa cause.

«Monsieur Maurice » comprend alors qu'il est en passe de perdre un soutien indispensable au maintien de son pouvoir. Loin de ses discours enflammés, il fait désormais profil bas : «Permettez-moi d'abord de vous faire part de mon profond et douloureux regret de l'inévitable malentendu, créé dans mes relations avec l'Église de Haute-Volta par mon mariage. Vous ne pouvez imaginer un seul instant, Éminence, que j'aie pu agir par provocation à l'égard des autorités religieuses $»^{53}$. Se sentant alors menacé par l'officialisation de l'hostilité du pouvoir catholique, Yaméogo cherche à ménager ses relations avec le cardinal Zoungrana. Cependant, malgré ses efforts diplomatiques, la «parenté ecclésiastique » du président de la République semble définitivement reniée et l'ancien séminariste devient le spectateur impuissant de la vindicte de l'Église. Le cardinal participant au concile Vatican II, c'est son vicaire qui, sur

52. Entretien avec Charles Bila Kaboré, Ouagadougou, 15 juin 2009.

53. Maurice Yaméogo à Mgr Zoungrana, Ouagadougou, 3 novembre 1965, CNABF, Cart. 6 V16. 
la base des principes humanistes précédemment occultés par l'épiscopat voltaïque, porte en public la critique de l'Église. On comprend alors la réaction présidentielle :

\begin{abstract}
Puisqu'à l'occasion du $5^{e}$ anniversaire de notre Indépendance, vous avez eu l'amabilité de nous révéler dans votre discours, les carences du Gouvernement en même temps que les méthodes dictatoriales de notre Parti, et cela en présence du corps diplomatique tout entier, (...) permettez-moi aussi, en tant que Chef d'État, toute considération religieuse mise à part, de dire que votre accueil à la fois très froid et très discourtois, pour ne pas dire très méprisant, à mon égard, a frappé tout mon Gouvernement qui n'a pas manqué de s'en émouvoir (...). Je manquerai à mon devoir de chrétien en n'attirant pas votre bienveillante attention sur vos maladresses, dont vous ne soupçonnez pas les conséquences incalculables, au-dedans et au dehors, si vous devez persister dans une telle voie ${ }^{54}$.
\end{abstract}

Maurice Yaméogo l'a bien senti, les «conséquences incalculables » ne se font pas attendre : le 3 janvier 1966, il occupe pour la dernière fois le fauteuil présidentiel, au plus grand soulagement de l'Église voltaïque ${ }^{55}$. De l'aveu d'un politicien de l'époque, « personne ne l'a fait tomber, c'est lui-même qui s'est jeté par terre $\aleph^{56}$. En effet, après avoir condamné l'opposition politique à la soumission ou à la clandestinité, puis contrarié les chefferies par des mesures législatives mortifères et enfin muté ou limogé de nombreux ministres et cadres de la fonction publique, Yaméogo provoque sa propre chute en se mettant à dos la seule force qui lui était restée fidèle, l'Église. Aussi, lorsque le mouvement syndical lance un important mouvement de contestation suite à des mesures d'austérité budgétaire amputant de $20 \%$ le salaire des fonctionnaires, le chef de l'État ne peut obtenir du cardinal Zoungrana qu'un appel à « la paix et à la compréhension mutuelle », prononcé du bout des lèvres (Kabeya Muase 1989 : 78). Maurice Yaméogo compte sur le prélat pour déclarer la grève illégale, mais le représentant de l'épiscopat voltaïque a bien compris la leçon : l'Église ne doit-elle pas éviter toute ingérence dans les affaires politiques?

\title{
Conclusion
}

« Je prie Dieu de me protéger de mes amis, mes ennemis je m'en charge $»^{57}$. C'est avec ces mots que le premier président de la République voltaïque comprend que la sympathie de l'Église catholique peut être autant bénéfique que nuisible à son projet personnel et national. En effet, même s'il a su contenir la contestation de ses opposants, il sait qu'il ne peut contrôler une instance qui, garante d'une autorité supra-politique, dispose d'une réelle indépendance au sein de la sphère publique. Certes, discours et cérémonies semblent de part et d'autre construire l'illusion d'une situation concordataire. Cependant, l'examen d'autres dimensions de la réalité dévoile un constat bien plus équivoque.

54. Maurice Yaméogo au Vicaire général de l'archevêché de Ouagadougou, Ouagadougou, 12 décembre 1965, CNABF, Cart. 6V16.

55. «On a chuté et je crois que l’Église a dû dire "Ouf” ». Entretien avec Charles Bila Kaboré, Ouagadougou, 15 juin 2009.

56. Entretien avec Henri Guissou, Ouagadougou, 23 juin 2009.

57. Maurice Yaméogo à Mgr Zoungrana, Ouagadougou, 19 août 1963, CNABF, Cart. 6 V16. 
Une première impression nous donne l'image d'une autorité ecclésiale presque subordonnée au régime de la Première République. Portant des vues théocratiques illusoires, elle semble à la peine pour faire valoir un cléricalisme finalement peu conforme aux prétentions autocratiques de Yaméogo. Cette impression de subordination peut être inversée en constatant le rapport d'engendrement qui lie l'État à la sphère ecclésiale. L'Église catholique profite alors de l'enfantement symbolique du politique pour renforcer sa visibilité aux côtés des dirigeants du pays. Toutefois, ne poussant pas la relation jusqu'à l'inceste, elle décide d'officialiser sa rupture avec le régime à partir du divorce du chef de l'État, moment où toute la population constate ouvertement que " Maurice a gâté ses relations avec 1'Église $»^{58}$. La hiérarchie catholique choisit alors de se révéler moralement dogmatique après avoir été politiquement pragmatique.

En outre, malgré les apparences, des dissensions se remarquent en filigrane dès l'avènement de la Haute-Volta indépendante. L'observation méticuleuse permet de distinguer dans les manières locales de faire de la politique une situation d' " extraterritorialité morale ", où prend place « un îlot de liberté de pensée et d'expression (...) au milieu d'une mer entièrement soumise au discours officiel $\|^{59}$. Habitée par des objectifs divers et composée d'éléments disparates, l’Église catholique voltaïque peut autant multiplier les accointances que les distances avec le pouvoir en place. Pour comprendre cette diversité, l'étude des caractéristiques sociologiques de l'institution catholique s'avère essentielle. En effet, entre un cardinal, un membre de la classe sacerdotale ouagalaise, un aumônier d'Action catholique, un missionnaire français ou un prêtre de paroisse rurale, les attitudes divergent. Nous sommes alors tentés de valider l'hypothèse formulée par G. André et M. Hilgers à la suite de Bourdieu et selon laquelle « un groupe occupant une position dominante a tendance à produire une idéologie religieuse spécifique qui renforce les appareils du pouvoir et les légitime tandis que les groupes dominés, aménagent, contournent, résistent et tentent de construire des "contre-hégémonies" " (André et Hilgers 2009 : 13). Il existerait ainsi au sein du milieu catholique voltaïque ce qu'A. Mélice a pu constater au Congo avec le kimbanguisme postcolonial : d'une part, une institution officielle tissant des liens étroits avec l'État et, d'autre part, un mouvement populaire développant une certaine propension à la critique du pouvoir (Mélice 2009).

Pour autant, il serait excessif de ne voir dans le comportement politique des acteurs religieux que des agissements de « classe». En effet, certaines homologies structurelles perdent parfois de leur valeur devant l'émergence de nouveaux contextes. Ainsi, il ne faut pas exclure de l'analyse l'étude des éléments conjoncturels : la recherche, suite à l'indépendance, d'une concorde nationale et d'une communauté d'intérêts, l'interdépendance socioéconomique et la diversion de Vatican II peuvent expliquer le silence officiel de l'Église. À l'inverse, les mesures autoritaires du régime, les inconduites de Yaméogo et le renversement de l'opinion publique mènent progressivement à une opposition de plus en plus affichée. En définitive, la plasticité des comportements politiques résulte ici autant de l'hétérogénéité du groupe catholique que du pragmatisme ecclésial, et nous ramène au constat de l'existence de cette « pluralité des modes d'intervention du religieux en politique » qu'évoquaient Constantin et Coulon (Constantin et Coulon 1997 : 13).

58. Entretien avec Zoubi Yaméogo, Ouagadougou, 2 mai 2010.

59. Expression de George Weigel citée par Philpott, 2008 : 153. 


\section{Références citées}

Afrique Nouvelle, 882, 3 au 9 juillet 1964.

André, Géraldine et Mathieu Hilgers, 2009. « Entre contestation et légitimation. Le religieux en contextes semiautoritaires en Afrique », Civilisations 58 (2) : 9-20.

BAyArt, Jean-François, 1981. « La politique par le bas en Afrique noire », Politique africaine 1 : 53-82.

, 1989. « Avant-propos : Églises africaines et contraintes économiques », Politique africaine 35 : 1.

1993. «Les Églises chrétiennes et la politique du ventre », in Jean-François Bayart (éd.), Religion et modernité politique en Afrique noire. Dieu pour tous et chacun pour soi, 129-160. Paris : Karthala.

Beucher, Benoît, mars 2009. «La naissance de la communauté nationale burkinabè ou comment le Voltaïque devint un "Homme intègre" ", Sociétés politiques comparées. Revue européenne d'analyse des sociétés politiques 13, http://www.fasopo.org/reasopo/n13/n13_article.pdf.

Bouncoungou, André-Jules, 1971. L'autorité chez les Mossi de Haute-Volta, mémoire de maîtrise : Institut Catholique de Paris.

Bourdieu, Pierre, 1971. « Genèse et structure du champ religieux », Revue française de sociologie 12 (3) : 295 334.

2001. Langage et pouvoir symbolique. Paris : Seuil.

Bouron, Jean-Marie, 2010a. « Les Pères Blancs, acteurs du jeu colonial. Mission catholique et enjeux politiques en Haute-Volta au lendemain de la seconde Guerre mondiale », Histoire et Missions chrétiennes 14 : 59-81.

2010b. «Indépendance des élites voltaïques, indépendance du pays : la mission catholique entre deux feux (1950-1960) », in Caroline Sappia et Olivier Servais (éds), Mission et engagement politique après 1945. Afrique, Amérique latine, Europe, 153-168. Paris : Karthala.

2010c. «Etre catéchiste en Haute-Volta à la fin de la période coloniale. Affirmation d'un personnage prosélyte, transformation d'une personnalité sociale », Social Sciences and Missions 23 (2) : 187-227.

Carrefour Africain 45, 18 février 1962.

Compaoré, R. A. Maxime, 1995. L'école en Haute-Volta : une analyse de l'évolution de l'enseignement primaire de 1947 à 1970, thèse de doctorat, Université Paris VII.

Constantin, François et Christian Coulon, 1997. «Religion et démocratie. Introduction à une problématique africaine ", in François Constantin et Christian Coulon (éds), Religion et transition démocratique en Afrique, 9-26. Paris : Karthala.

Coulon, Christian, 1991. " Religions et politique », in Christian Coulon et Denis-Constant Martin (éds), Les Afriques politiques, 87-105. Paris : La Découverte.

DE BENOIST, Joseph-Roger, 1987. Église et pouvoir colonial au Soudan français, administrateurs et missionnaires dans la Boucle du Niger (1885-1945). Paris : Karthala.

Djereke, Jean-Claude, 2001. L'engagement politique du clergé catholique en Afrique noire. Paris : Karthala.

Dupont, André (Mgr), s.d. Histoire religieuse de la Haute-Volta des débuts à 1971. s.1., dactylographié.

Gallibert, Didier, 2010. « La mission postcoloniale entre notoriété et précarité. Etude comparée de deux expulsions de jésuites à Madagascar », in Caroline Sappia et Olivier Servais (éds), Mission et engagement politique après 1945. Afrique, Amérique latine, Europe, 289-300. Paris : Karthala.

Gocking, Roger, 2005. The history of Ghana. Westport : Greenwood Press.

Guirma, Frédéric, 1991. Comment perdre le pouvoir? Le cas de Maurice Yaméogo. Paris : Chaka.

Hastings, Adrian, 1979. A history of African Christianity. 1950-1975. Cambridge : Cambridge University Press. 
Kabeya Muase, Charles, 1989. Syndicalisme et démocratie en Afrique Noire. L'expérience du Burkina Faso. Abidjan et Paris : Inadès et Karthala.

La Croix, 27 avril 1962.

Les Evêques de Haute-Volta, 1959. Le chrétien dans la cité. Problèmes politiques, problèmes économiques, problèmes sociaux. Bobo-Dioulasso : Savane.

Martin, Denis-Constant, 1991. « Les cultures politiques », in Christian Coulon et Denis-Constant Martin (éds), Les Afriques politiques, 157-171. Paris : La Découverte.

Mвемвe, Achille, 1988. Afriques indociles. Christianisme, pouvoir et État en société postcoloniale. Paris : Karthala.

Melice, Anne, 2009. «Le kimbanguisme et le pouvoir en RDC entre apolitisme et conception theologicopolitique », Civilisations 58 (2) : 61-79.

Nkay MaLu, Flavien, 2003. « L'Église catholique, le peuple et l'État dans la République démocratique du Congo (1960-1990) », in Philippe Delisle et Marc Spindler (éds), Les relations Églises-État en situation postcoloniale. Amériques, Afrique, Asie, Océanie XIXe-XXe siècles, 277-307. Paris : Karthala.

OтAYек, René, 1991. «Organisations et compétitions politiques », in Christian Coulon et Denis-Constant Martin (éds), Les Afriques politiques, 186-199. Paris : La Découverte.

-_-_, 1997. « L'Église catholique au Burkina Faso : un contre-pouvoir à contretemps de l'histoire ? », in François Constantin et Christian Coulon (éds), Religion et transition démocratique en Afrique, 221-258. Paris : Karthala.

Philpott, Daniel, 2008 [2005]. « La vague catholique », in Larry Diamond et al., Les religions du monde et la démocratie, 147-165. Paris : Nouveaux Horizons et ARS.

Rivière, Claude, 1997. « Religion et politique en Afrique noire », Anthropos 92 (1/3) : 21-34.

Somé, Magloire, 2001. « Christian Base Communities in Burkina Faso : Between Church and Politics », Journal of Religion in Africa 31 (3) : 275-304.

YAmÉogo, Maurice, 1938. « Récit de Maurice Yamwaogho, élève de quatrième à Pabré », Grands Lacs : $163-164$. 